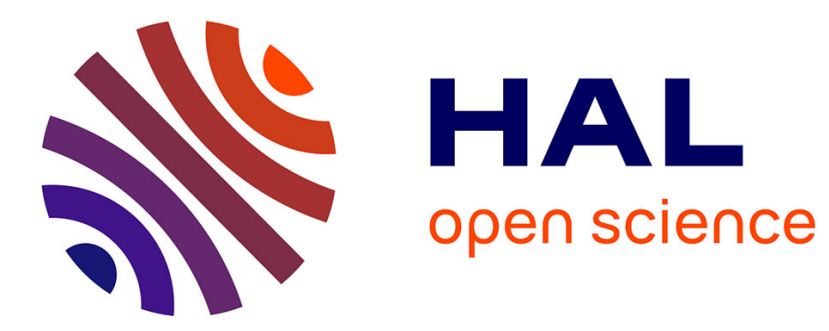

\title{
Fernand Oury, les groupes de la classe coopérative et l'approche psychanalytique (1955-1958)
}

\author{
Arnaud Dubois, Patrick Geffard
}

\section{To cite this version:}

Arnaud Dubois, Patrick Geffard. Fernand Oury, les groupes de la classe coopérative et l'approche psychanalytique (1955-1958). Cliopsy, 2015. hal-01898278

\section{HAL Id: hal-01898278 \\ https://hal.science/hal-01898278}

Submitted on 18 Oct 2018

HAL is a multi-disciplinary open access archive for the deposit and dissemination of scientific research documents, whether they are published or not. The documents may come from teaching and research institutions in France or abroad, or from public or private research centers.
L'archive ouverte pluridisciplinaire HAL, est destinée au dépôt et à la diffusion de documents scientifiques de niveau recherche, publiés ou non, émanant des établissements d'enseignement et de recherche français ou étrangers, des laboratoires publics ou privés. 


\title{
Fernand Oury, les groupes de la classe coopérative et l'approche psychanalytique (1955-1958)
}

\author{
Arnaud Dubois et Patrick Geffard
}

«Il faudrait travailler sur les groupes dans les classes / j'y connais rien / les phénomènes de groupe dans les classes / ce qui rend les gens fous c'est pas tellement des histoires inconscient [sic] / pas seulement des histoires inconscientes ».

Fernand Oury (propos recueillis par M. Amram et F. d'Ortoli, 2010)

\section{Introduction}

Cet article présente les premiers résultats d'une recherche que nous menons depuis deux ans pour tenter d'apporter des éléments de réponse à la question suivante : pourquoi la pédagogie institutionnelle a-t-elle si peu emprunté à la psychanalyse groupale, pourquoi s'en est-elle si peu inspirée ? Au cours d'une précédente recherche, il nous est en effet apparu que les références psychanalytiques mobilisées dans le champ de la pédagogie institutionnelle proviennent rarement de l'approche psychanalytique groupale (Dubois et Geffard, 2013). À titre d'exemple, examinons la rubrique «groupes, sociométrie, psychodrame, nondirectivité... » qui fait partie de l'annexe intitulée « livres utiles » publiée à la fin du livre De la classe coopérative à la pédagogie institutionnelle (Oury et Vasquez, 1971, p. 760 à 768). Sur les 153 ouvrages signalés, 12 titres seulement appartiennent à cette rubrique et ils abordent essentiellement la pratique du psychodrame ou de la sociométrie. Recherches sur les petits groupes, de Wilfred Ruprecht Bion, est bien référencé, mais il est très peu utilisé dans le corps de l'ouvrage. Pourquoi une telle distance alors que la pédagogie institutionnelle prétend s'intéresser aux processus inconscients à l'œuvre dans les classes et que celles-ci sont considérées comme «à la fois un groupe et un ensemble de groupes » (Oury et Vasquez, 1971, p. 689) ?

Une réponse « de premier niveau » pourrait se trouver dans le lien entre la pédagogie institutionnelle et la pensée lacanienne. Ce lien s'incarne notamment dans la relation fraternelle entre Fernand Oury (1920-1998), instituteur, et Jean Oury (1924-2014), psychiatre et psychanalyste proche 
de Jacques Lacan avec lequel il a été en analyse de 1953 à 1981 . On connaît la dimension équivoque des propos de Lacan sur la question du groupe - nous y reviendrons en fin d'article. Il n'est pas impossible que son faible intérêt pour cette question ait contribué à ce qu'elle soit peu prise en charge dans le champ de la pédagogie institutionnelle.

Le développement et la diffusion de la psychanalyse groupale en France peuvent être datés des années 1956-1960, notamment avec les travaux de Didier Anzieu (1923-1999), qui publie en 1956 Le psychodrame analytique chez l'enfant, suite à un article inaugural de Serge Lebovici, René Diatkine et Évelyne Kestemberg (1952). Le Cercle d'études françaises pour la formation et la recherche, approche psychanalytique du groupe, du psychodrame, de I'institution (Ceffrap) est fondé en 1962 par Didier Anzieu et les premiers travaux de Wilfred Ruprecht Bion sont traduits en français à partir de 1965. Les recherches psychanalytiques sur les groupes et le développement des pratiques groupales d'orientation psychanalytique sont donc contemporains de la naissance de la pédagogie institutionnelle. On pourrait alors penser que la méconnaissance signalée plus haut est une conséquence d'apparitions conjointes qui n'ont pas permis que l'approche pédagogique s'étaye vraiment sur des travaux qui en étaient eux-mêmes à leurs débuts.

Malgré ce contexte, il nous semble pourtant que la relative distance avec la question du groupe dans le champ de la pédagogie institutionnelle est antérieure au développement de cette pédagogie comme du lacanisme en France. Il nous est même apparu que des éléments de réponse à la question que nous nous posions pouvaient être antérieurs à la naissance de ce mouvement pédagogique. Une relative appréhension à l'égard des questions groupales n'était-elle pas présente dans la pensée de Fernand Oury en amont de cette fondation, à une époque où il était encore engagé dans le mouvement Freinet ? Pour avancer sur cette question, nous avons analysé les premiers textes publiés par Fernand Oury pour tenter de percevoir si ces premiers écrits ne contenaient pas des éléments de son rapport au groupe qui se seraient par la suite transmis au sein du courant pédagogique qu'il a si fortement contribué à fonder.

Avant de présenter le corpus sur lequel porte notre travail, il nous faut donner quelques indications sur nos liens à notre sujet de recherche. Nous mentionnerons notamment nos parcours de praticiens de la pédagogie institutionnelle dans l'enseignement élémentaire ou secondaire, notre participation à des groupes de travail animés par Fernand Oury ou celles et ceux qu'il a inspirés et le fait que nous avons été, l'un comme l'autre, fondateurs de groupes locaux de pédagogie institutionnelle. Nous avons partiellement rendu compte de l'élaboration de nos mouvements transférentiels respectifs dans des travaux antérieurs (Dubois, 2011 ; Geffard, 2010), ceux-ci ne pouvant être repris de manière détaillée dans le cadre de cet article. 


\section{Les premiers textes de Fernand Oury rédigés entre 1955 et 1958}

\section{Limites chronologiques}

Pour cette recherche, nous avons choisi d'analyser les premiers articles écrits par Fernand Oury entre 1955 et 1958. Une précédente recherche (Dubois, 2011) sur les premières monographies rédigées par Fernand Oury a permis de rassembler environ cent dix textes publiés entre 1955 et 1996. Pour démarrer cette recherche-ci, nous avons fixé comme début de limite chronologique le mois de juin 1955, date du premier écrit publié par Fernand Oury que nous avons trouvé.

Nous avons choisi l'année 1958 comme borne chronologique terminale parce que nous voulions examiner la période antérieure à la naissance de la pédagogie institutionnelle. Jean Oury, frère cadet de Fernand et fondateur de la clinique de La Borde en 1953, a dit avoir proposé l'expression « pédagogie institutionnelle » au cours du congrès du mouvement Freinet qui s'est tenu à Paris en avril 1958. Ce congrès est un moment clé dans le développement de ce qui devient, à partir de 1961, un courant pédagogique distinct du mouvement Freinet. En effet, il a été organisé par l'Institut parisien de l'école moderne, groupe parisien de l'Institut coopératif de l'école moderne, dont font partie Raymond Fonvieille (1923-2000) et Fernand Oury. Dans un article publié en mars 1958, Raymond Fonvieille écrit : «un peu d'inconscience au départ, il en faut quand on prend la charge de l'organisation d'un congrès » (Fonvieille, 1958, p. 3). Il précise que l'organisation de ce congrès à Paris devait «permettre aux milieux officiels de la capitale de s'informer de ce que sont vraiment les techniques Freinet » (Ibid.). Pour lui, un des enjeux de ce congrès était d'organiser une confrontation entre «Freinet, [...] des grands noms de la recherche pédagogique [et le] monde de la psychologie et de la médecine » (Ibid.). Cette « rencontre » a été organisée au cours de la soirée du 3 avril pendant laquelle sont intervenus successivement des psychanalystes - notamment Georges Mauco, Xavier Audouard et Jean Oury - et des instituteurs, dont Célestin Freinet (1896-1966) et Fernand Oury ${ }^{1}$.

Cette première rencontre entre les membres du mouvement Freinet et des représentants de la sphère «psy » (Carroy, 2005, p. 204) marque le point de départ du développement de ce qui devient, à partir de 1964, le courant psychanalytique de la pédagogie institutionnelle (Dubois, 2011). Ce congrès de 1958 est important aussi dans la mesure où il témoigne de l'influence grandissante du groupe parisien de I'Institut coopératif de l'École moderne (ICEM) qui aboutit, en 1961, à l'expulsion d'une partie des Parisiens (Fonvieille, 1989 ; Lamihi, 1994 ; Savoye, 2004).

\section{Présentation du corpus}

Entre juin 1955 et avril 1958, Fernand Oury a publié douze articles dont nous n'avons retenu que ceux dans lesquels le terme «groupe » apparaît. Nous avons ainsi constitué un corpus de six articles publiés dans trois
1. Oury, F. (1958, 3 avril). Compte-rendu de la conférence prononcée au congrès de Paris. Document dactylographié conservé dans les archives de F. Oury à l'école de la Neuville, carton 1/17. (p. 5). 
revues: L'Éducateur (Oury, 1955b et d; 1957c) ; L'Éducateur d'île-deFrance (Oury, $1957 f$ et 1958b); un article a été publié à la fois dans L'Éducateur (Oury, 1957a) et la Revue pratique de psychologie, de la vie sociale et d'hygiène mentale (Oury, 1957d). À l'exclusion du dernier, ces périodiques font partie de la presse d'éducation et d'enseignement, expression qui recouvre «tous les périodiques traitant d'éducation, de formation et d'enseignement » (Caspard et Caspard-Karydis, 2006, p. 563).

Que sont ces trois revues ? Célestin Freinet est le fondateur de L'Éducateur dont il dirige la publication jusqu'à sa mort en 1966. Ce titre qui paraît à partir d'octobre 1939 fait suite à L'Éducateur prolétarien, publié d'octobre 1932 à juillet 1939 et également fondé par Freinet. Sous le titre générique de L'Éducateur, la revue a connu des éditions multiples, soit sous la forme d'une scission de la revue elle-même en deux titres différents, soit sous la forme de suppléments intégrés dans la revue. Elle est éditée à Cannes, siège de I'ICEM qui est l'organe pédagogique du mouvement à partir de 1950. L'Éducateur est associé à d'autres périodiques relevant de la pédagogie Freinet, notamment les parutions régionales des groupes départementaux de I'ICEM, comme L'Éducateur d'île-de-France, qui est la publication du groupe francilien du mouvement Freinet. Cette revue, dans laquelle Raymond Fonvieille et Fernand Oury sont très actifs, est publiée à partir de 1957 et obtient rapidement le soutien de Roger Gal (1906-1966), alors directeur de I'Institut pédagogique national (IPN). Favorable au développement des mouvements pédagogiques à cette époque, il utilise les moyens de I'IPN pour prendre en charge le tirage et la diffusion de L'Éducateur d'île-de-France. Le développement de ce périodique sera en partie à l'origine des tensions entre le groupe parisien et le mouvement Freinet à partir de 1960 .

La Revue pratique de psychologie, de la vie sociale et d'hygiène mentale, créée en juin 1953 et animée par Pierre Doussinet, co-fondateur avec Alice Delaunay de la Fédération d'Aide à la Santé Mentale (FASM) Croix-Marine, ne relève pas a priori de la presse d'éducation. Dans les années 1950 , elle joue un rôle d'interface entre psychiatres et pédagogues. On peut y lire des textes pédagogiques, notamment sur ce qu'on appelle alors l'enfance inadaptée, ainsi que des articles de psychiatrie ou de psychanalyse (André Berge, Clément Launay,...).

\section{Quelques éléments de la biographie de Fernand Oury}

Après avoir indiqué les limites chronologiques de notre étude et le corpus sur lequel a porté notre analyse, nous allons maintenant présenter l'auteur des articles au moment où il les a rédigés. En ayant recours à des sources de différentes natures, nous avons rassemblé ici quelques éléments de la biographie de Fernand Oury avant 1958, susceptibles d'éclairer, au moins partiellement, son rapport au groupe. 


\section{Archives, sources imprimées et orales}

Nous avons pris appui sur une série de documents issus de fonds d'archives publics et privés, rassemblés pour une précédente enquête historique (Dubois, 2011), que nous avons complétée par de nouvelles recherches. Nous avons consulté les archives de Fernand Oury actuellement conservées à l'école de la Neuville, regroupant essentiellement des textes manuscrits ou ronéotypés ainsi que des articles, pour la période qui nous intéresse. Nous avons fait une recherche systématique dans ce fonds constitué par Fernand Oury lui-même. Ce fonds d'archives privé constitue un ensemble documentaire d'une grande richesse. Nous avons mené d'autres recherches dans des fonds d'archives publics - archives du rectorat de Paris, archives du département de la Seine à Paris - cherchant, en vain, le dossier d'instituteur de Fernand Oury. Nous avons aussi consulté les archives du Centre national de pédagogie spéciale (CNPS) de Beaumont-sur-Oise, conservées aux archives de Versailles, les archives de la ville de Nanterre où Fernand Oury a enseigné, ainsi que les actes d'État civil le concernant. Le temps nous a manqué pour consulter les archives de Raymond Fonvieille, récemment déposées à I'Université Paris 8 , et dont le dépouillement promet d'enrichir cette histoire.

Parmi les sources imprimées, il faut signaler en particulier le seul texte autobiographique publié par Fernand Oury, ses « Mémoires d'un âne » dans lesquelles il retrace son parcours avant 1949 et ses débuts dans le métier d'instituteur (Oury et Vasquez, 1971, p. 49 à 229). Le témoignage publié par Raymond Fonvieille, instituteur qui a participé à la naissance de la pédagogie institutionnelle, est quant à lui essentiel pour saisir la chronologie des événements, les enjeux et le rôle de chacun dans cette histoire (Fonvieille, 1989 et 1998). Il apporte quelques précieux éléments sur la biographie de Fernand Oury.

Outre les documents d'archives et les sources imprimées, nous avons « inventé » (Voldman, 1993, p. 125) une partie de nos sources en menant des entretiens avec plusieurs témoins ayant été plus ou moins proches de Fernand Oury : Jean Di Rosa, compagnon de Fernand Oury, qu'il a rencontré en 1942 en étant son élève ; André Inizan, qui a enseigné au CNPS où Fernand Oury a été son élève; Michel Faligand, instituteur militant du mouvement Freinet et membre de I'Institut Parisien de l'École Moderne (IPEM) avec Raymond Fonvieille et Fernand Oury ; Joëlle et François Oury, deux de ses trois enfants, dont le second a été scolarisé dans la classe de son père en CP en 1952-53 à La Garenne-Colombes.

Quelques recherches nous ont été utiles dans ce travail biographique. Le livre publié par Raymond Bénévent et Claude Mouchet (2014) est venu combler un manque dans la bibliographie, puisque jusqu'alors peu de textes biographiques avaient été consacrés à Fernand Oury et aucun n'était aussi complet. Quelques travaux d'historiens permettent de mieux connaître le contexte des années de formation de Fernand Oury, notamment sa relation avec Félix Guattari (Dosse, 2007). 
Il s'agit pour nous de tenter de formuler ici des hypothèses concernant le rapport au groupe de Fernand Oury, en tentant de mettre en lumière quelques expériences groupales telles qu'elles peuvent nous apparaître à partir de ces différentes sources et travaux. Nous reprendrons la distinction devenue classique entre groupes primaire et secondaire (Anzieu et Martin, 1968 , p. 38). Cette distinction permet de suivre la chronologie de la trajectoire biographique de Fernand Oury. Sur le plan méthodologique, cette tentative d'éclairer un parcours biographique en ayant recours à des concepts psychanalytiques relève de ce qu'Henry Rousso qualifie « d'opération métabiographique » (2002, p. 128), initiée par Freud luimême, notamment dans L'homme Moïse et la religion monothéiste (1939) ou la biographie du président Wilson, publiée avec William Bullitt (1966). Cette voie a été empruntée aussi par Georges Devereux (1995) dont le travail historique a occupé une grande part des recherches.

\section{Le groupe d'appartenance primaire}

La famille est le premier groupe d'appartenance, le «groupe originaire » (Lecourt, 2008, p. 24). Jean-Claude Rouchy considère «que le groupe familial constitue essentiellement le groupe d'appartenance primaire [et que] les groupes naturels auxquels celui-ci est relié peuvent en faire partie et le traversent (appartenance religieuse, classe, ethnie) 》 (Rouchy, 2009, p. 151). Pour lui, «le groupe d'appartenance primaire est donc beaucoup plus large que la famille nucléaire : il comprend aussi des oncles et tantes, des cousins et cousines proches, des amis avec lesquels I'on passe souvent des moments ou des vacances, des voisins avec lesquels se sont établies des relations de proximité dans le quotidien » (Ibid.). Nous allons tenter de dessiner ici, à grands traits, le « groupe d'appartenance primaire » dans lequel est né Fernand Jean Oury, le 18 janvier 1920, boulevard du Port Royal, à Paris.

Il est le premier fils de Georges Alphonse Oury et Léontine Henriette Lecler, âgés respectivement de 36 et 28 ans à cette date. Par sa naissance, Fernand Oury fait advenir sa famille nucléaire comme groupe, dans la mesure où un groupe comporte au moins trois personnes (Anzieu et Martin, 1968 , p. 44). Comme le soulignent Bénévent et Mouchet, Fernand Oury est né dans « une famille éprouvée par la guerre » (2014, p. 24). Son père, alors polisseur à l'usine Hispano-Suiza de Bois-Colombes, a perdu « au front ses trois frères : Jean, Fernand et Gaston » (Ibid.). Fernand Oury porte donc deux prénoms d'oncles qu'il n'a pas connus. Du côté paternel, ses grandsparents, René Oury (1856-1894) et Émilie Grapinet (1855-1902) sont décédés eux aussi avant sa naissance. Son grand-père maternel, disparu en novembre 1921, I'a à peine connu. La mort de celui-ci a beaucoup affecté sa fille Léontine, qui « adorait son père et a fait une dépression, alors que [...] Fernand était tout petit et, d'après ce qu'il en disait adulte [il aurait fait] une 
De cette génération, sa grand-mère maternelle, Eugénie Bouyé (1866-1948) est la seule qui était présente auprès du petit Fernand. Elle a participé à son éducation en venant aider sa fille pour l'élever, lui et ses frères cadets ${ }^{3}$, Paul (1922-1985) et Jean (1924-2014). En 1926, elle s'installe avec le reste de la famille dans le pavillon acquis à La Garenne-Colombes par Léontine Oury qui crée alors une petite agence immobilière. La vie de Fernand Oury a été fortement marquée par la mort, le deuil et la dépression. D'abord en tant qu'enfant, au sein de son «groupe d'appartenance primaire », lors des décès de ses oncles et de son grand-père (la mort de ce dernier semblant avoir eu sur lui un profond retentissement, transmis par sa mère), et plus tard en tant que père, comme nous allons le voir.

Fernand Oury est par ailleurs né dans une famille dans laquelle le matriarcat était dominant : son père était assez « effacé » et « Fernand semble avoir lui-même assumé à l'égard de ses frères une part de la fonction paternelle » (Bénévent et Mouchet, 2014, p. 25-26). Sa mère et sa grand-mère maternelle occupaient une place dominante dans cette structure familiale. Le témoignage de Jean Oury, recueilli par Bénévent et Mouchet, comme celui de Jean Di Rosa que nous avons sollicité, ainsi que les recherches de François Dosse sur la jeunesse de Félix Guattari convergent pour dessiner le portrait d'un enfant et d'un adolescent qui participait à des groupes dans lesquels il semble occuper le plus souvent la place de l'aîné. Dans la banlieue ouvrière qu'est alors La Garenne-Colombes, le terrain vague est un terrain de jeu pour ces groupes d'enfants dont les frères plus jeunes, Paul et Jean, sont membres.

\section{Les groupes d'appartenance secondaire}

Adolescent, puis adulte, Fernand Oury a appartenu à de nombreux groupes secondaires, au sens de ces «groupes institués au sein desquels des individus sont réunis [et qui sont] fort différents des groupes primaires, car leur constitution présuppose que l'individuation soit suffisamment avancée pour qu'existent une relation d'objet et des rapports d'individu à individu » (Rouchy, 2009, p. 153).

Fernand Oury rejoint, puis anime, différents groupes dans le cadre des mouvements de jeunesse qui se sont développés en France dans les années 1930, notamment sous I'influence du Front populaire. À partir de 1936, il participe aux activités du mouvement des auberges de jeunesse en tant qu'usager. Lors des vacances, il part camper en groupe, avec d'autres jeunes de La Garenne-Colombes, dans le cadre du Centre laïque des auberges de jeunesse (CLAJ). C'est par ce biais qu'il est en relation avec le mouvement trotskiste. À l'été 1944, alors que la France est encore en guerre, il est «animateur du patronage laïque $»^{4}$ et encadre les loisirs des jeunes de La Garenne-Colombes. À la Libération, il a des responsabilités dans I'animation des auberges de jeunesse et retrouve Di Rosa et Guattari qu'il a eus comme élève pendant quelques semaines en 1942-1943. Tous deux participent à un séjour encadré notamment par Fernand Oury dans le
3. Entretien avec Joëlle Oury, le 10 mai 2011.

4. Entretien avec Jean Di Rosa, le 16 août 2007. 
5. Lettre manuscrite de Jean Di Rosa, le 11 novembre 2009.

6. Archives de Paris. Relevés de suppléance sous la cote Pérotin/40125/75/13228.

7. Inscrit sous le $n^{\circ} \mathrm{d}^{\prime}$ ordre 247 , il est classé $102^{\mathrm{e}}$, ce qui ne lui permet pas d'être reçu. Archives de Paris. Dossiers du concours d'entrée de l'École normale. Carton 3433W68.

8. Les citations qui suivent sont extraites de l'entretien avec Joëlle Oury, le 10 mai 2011. cadre des caravanes ajistes, à Recloses en forêt de Fontainebleau, puis à Albiez-le-Vieux en Maurienne, en 1945-1946.

À partir de 1939, date à laquelle il devient instituteur, il encadre aussi des groupes d'élèves dans le cadre scolaire. Bachelier la même année, il entame des études de sciences physiques à l'université, mais les arrête rapidement ${ }^{5}$. Il devient alors instituteur suppléant dans l'enseignement privé à Paris ${ }^{6}$. Il échoue au concours d'entrée de l'École normale ${ }^{7}$, mais il est ensuite reçu au deuxième concours. Ce mode d'accès au statut d'instituteur, ouvert aux suppléants, comportait une épreuve pratique et dispensait de la formation en École normale. Fernand Oury obtient le certificat d'aptitude professionnelle (CAP) en 1941. Après une arrestation pour participation à la Résistance et une expérience du travail forcé « quelques mois à Toul ${ }^{8}$, il prend en charge une classe à l'école Ernest Renan de La Garenne-Colombes, rue Roussel, en 1944. Il y enseigne pendant dix ans, aux côtés de Denise, qu'il a épousée en janvier 1941.

Un nouveau groupe familial se forme avec elle et leur premier fils Sylvain, né en octobre 1943. Malheureusement, cet enfant meurt prématurément des suites d'une pneumonie en janvier 1944. Fernand Oury se confronte à nouveau au deuil, qu'il vit « comme une sorte de malédiction ». Le couple est effondré et la naissance de Joëlle un an plus tard, le 25 le janvier 1945, recrée un groupe familial, élargi par les naissances de François en juin 1946, puis de Frédéric en janvier 1952. La famille vit rue Roussel et François est scolarisé dans la classe de son père. Frédéric avait une santé fragile : il semble qu'il ait fait « une encéphalite à l'âge d'un an suite à un vaccin antivariolique ». Plus tard, il attrape «la tuberculose à l'âge de six ans », ce qui l'oblige à quitter le groupe familial pour passer une année dans un aérium. Il en serait revenu « ralenti » et « un peu dans la lune ». Son entrée à l'école a ainsi été retardée d'un an. D'après le témoignage de Joëlle Oury, il s'agit de l'année 1958-1959, qui a été très éprouvante pour Fernand Oury, puisque c'est aussi l'année du décès de son père, le 13 avril. Plus tard, à l'âge de 19 ans, «Frédéric a complètement décompensé dans la schizophrénie ». Pour Joëlle Oury, c'est «la grande blessure de [Fernand Oury], c'est son talon d'Achille, son grand malheur. Il l'a pris dans sa classe pendant plusieurs années et avec tout son savoir et ses techniques [pédagogiques], tout ce qu'il a pu faire pour Frédéric [...] ça n’a pas marché ».

Pour Fernand Oury, le groupe familial a été marqué par des deuils précoces, comme nous l'avons vu plus haut lorsqu'il était enfant. Le deuil se renouvelle lorsqu'il devient père, confronté à la mort de son premier fils. En 1958, date à laquelle nous avons décidé d'arrêter notre étude, si l'état de santé de Frédéric ne permet évidemment pas de connaître ce qui adviendra plus tard, sans doute Fernand Oury a-t-il été très éprouvé par la maladie de son fils.

En même temps qu'il anime et encadre des groupes d'enfants - en tant qu'animateur puis instituteur - il crée un nouveau groupe familial et 
participe à des groupes d'adultes qu'il contribue à animer, notamment dans le mouvement initié par Freinet. Sa rencontre avec ce dernier, en septembre 1949, a été décisive dans son parcours. Il rejoint alors le groupe parisien des instituteurs du mouvement Freinet qui fonctionne depuis 1946. Ce groupe se structure autour de Raymond Fonvieille, par la création de I'IPEM dont les statuts sont déposés en 1952 (Fonvieille, 1989, p. 56). Fonvieille, instituteur depuis 1943, a été I'un des principaux animateurs du groupe parisien du mouvement Freinet entre 1952 et 1961, dans lequel Fernand Oury est progressivement de plus en plus actif.

En 1954, Fernand Oury est en formation pendant six mois au Centre national de pédagogie spéciale de Beaumont-sur-Oise (Oury et Vasquez, 1967 , p. 259 ; Oury et Vasquez, 1971, p. 174). Il est admis au certificat d'aptitude à l'enseignement des enfants arriérés (CAEA) à la session de janvier 1955, puis prend en charge une classe de perfectionnement à l'école Paul Langevin de Nanterre en octobre. C'est dans cette classe que son fils Frédéric a été scolarisé à partir de 1959. Au cours de sa formation, Fernand Oury était très impliqué dans les groupes d'instituteurs en formation, notamment engagé sur le plan syndical ${ }^{9}$.

Au terme de cette courte enquête historique, il apparaît que Fernand Oury a appartenu jusqu'en 1958 à de nombreux groupes qu'il a lui-même contribué à former et/ou à animer. C'est le cas de la plupart des «groupes secondaires » dont il a été membre : il a très souvent eu une position d'animateur dans ces groupes, que ce soit avec des groupes de jeunes dans le cadre de leurs loisirs ou avec des groupes d'élèves en tant qu'instituteur. Dans les groupes d'adultes, il a souvent adopté une position analogue d'animateur, notamment aux côtés de Raymond Fonvieille au sein de I'IPEM. Or, des recherches antérieures menées auprès de praticiens de la pédagogie institutionnelle ont montré toute l'importance tenue par celui qui est en position $d^{\prime}$ « initiateur » et combien les propos actuels viennent s'étayer sur des paroles antérieures souvent présentées comme « fondatrices 》 (Geffard, 2010). Au-delà de ce que les acteurs du champ peuvent eux-mêmes repérer, on peut faire I'hypothèse que les sujets engagés dans ces pratiques pédagogiques sont aussi aux prises avec des processus de transmission qui n'appartiennent pas uniquement au registre du manifeste et du conscient.

\section{Les usages du mot « groupe » dans les premiers articles de Fernand Oury}

La présence du terme « groupe » dans les six articles étudiés est importante puisque I'on en trouve pas moins de 48 occurrences, auxquelles on peut ajouter le mot « groupement » rencontré une fois. Concernant cette époque, la polysémie du terme groupe dans les milieux qu'il fréquente et la labilité de son usage par Fernand Oury nous sont apparues remarquables.

Deux emplois principaux du mot se partagent 18 apparitions pour l'un et 17 pour l'autre. Dans le premier cas, il s'agit d'une utilisation visant à désigner
9. Entretien avec André Inizan, mai 2009. 
l'établissement scolaire alors que, dans le second, il est plutôt question d'évoquer soit le groupe-classe dans son ensemble, soit un sous-groupe institué dans la classe coopérative. La formulation employée ici fait évidemment écho à la distinction entre établissement et institution proposée très tôt par François Tosquelles (1912-1994), psychiatre d'origine catalane dont la pratique thérapeutique est à l'origine de la psychothérapie institutionnelle. Née dans les années 1940, cette différenciation vise à distinguer l'organisation émanant de la volonté publique centrale - l'État et les institutions localement mises en place par les professionnels qui ont reçu la charge des missions publiques (Tosquelles, 2014). Les institutions offrent des espaces de rencontres et d'interactions entre les différents participants à la vie de l'établissement qui ne se confondent pas avec les statuts « officiels» de chacun. $\mathrm{Si}$, à l'époque de la rédaction des articles considérés ici, Fernand Oury a déjà été en contact avec François Tosquelles, nous sommes pourtant encore loin d'une appropriation de la discrimination proposée par le psychiatre, puisque au fil des articles l'usage du mot groupe va renvoyer de manière un peu indistincte aux divers sens possibles.

On note pourtant une opposition sous-jacente entre le « groupe scolaire » et le «groupe-classe » lorsque celui-ci est entendu comme s'inscrivant dans des pratiques pédagogiques dénommées classe active, classe moderne ou encore classe coopérative: "Une classe active de 1955 a toute chance d'être isolée dans un groupe traditionnel » (Oury, 1955b, p. 26) ; «Dans un groupe scolaire, il est, malheureusement, d'autres dressages qu'il faut faire » (Id., p. 57) ; «La classe moderne est d'abord obligatoirement isolée dans un groupe » (Oury, 1957c, p. 17).

Les solutions envisagées pour résoudre cette opposition entre la classe pratiquant les techniques Freinet et le groupe scolaire urbain sont alors celles de la «création de petits groupes scolaires à l'échelle humaine permettant le travail "artisanal" en pédagogie » (Id., p. 20) ou la formation « d'équipes de travail œuvrant dans le même sens [...] soit à l'intérieur d'un groupe scolaire, soit dans un groupe expérimental » (Oury, 1958b, p. 4).

En lien avec ce qui a été dit plus haut sur son itinéraire biographique, nous notons que lorsque Fernand Oury appelle de ses vœux la formation d'équipes de travail, il indique que «ce sont les équipes qui ont permis les progrès pédagogiques dans les colonies de vacances » (Ibid.). Toutefois, il n'explicite ni les raisons de son choix du terme équipe par rapport à celui de groupe, ni en quoi et comment les équipes en question ont pu permettre certaines avancées.

Nous retrouvons d'ailleurs le recours au vocable équipe, en démarcation de celui de groupe, quand il est question des modalités d'organisation de la classe de type Freinet : "L'adaptation sociale se fait par les petits groupes. Petits groupes de travail variés et variables (peinture, imprimerie, nettoyage), mais surtout équipes permanentes et autonomes » (Oury, 1955b, p. 28). 
Là encore, des liens peuvent être faits par le lecteur d'aujourd'hui, mais ils seraient anachroniques. En effet, l'expression petits groupes de travail peut faire penser à la distinction proposée par W. R. Bion entre ce qu'il désigne comme le groupe sous l'emprise des hypothèses de base - basic assumption group (Bion, 1961) - et ce qu'il nomme le « groupe de travail » - work group (Id.). Ce dernier étant pour Bion le groupe qui commence à mettre des mots sur les émotions de base auxquelles la situation groupale renvoie les participants. Pour le psychanalyste anglais, «l'organisation et la structure sont les armes du groupe de travail », des armes qui lui permettent d'être moins facilement «submergé » par les hypothèses de base (Id.). Ce qui pourrait inviter à un rapprochement entre les deux auteurs, dans la mesure où certaines propositions de Fernand Oury s'expriment selon des formes proches de celles utilisées par Bion : «Plutôt que de vouloir en vain s'imposer une attitude fictive qui ne trompera pas longtemps les enfants, [l'enseignant] sera plus efficace par la structuration et par les institutions qu'il peut donner à ce groupe »(Oury, 1955b, p. 26).

Mais au moment de l'écriture des premiers articles de Fernand Oury, ce rapprochement n'est pas d'actualité. Recherches sur les petits groupes n'a pu influencer à ce moment-là la réflexion du pédagogue car l'ouvrage n'a été publié qu'en 1961 dans son édition originale et en 1965 dans sa traduction française. Et bien que Fernand Oury les mentionne entre 1955 et 1958, il n'est pas encore en mesure de penser avec précision les articulations et les oppositions entre le « groupe » à durée limitée, constitué en fonction d'une tâche particulière, et l'« équipe » organisée à partir du sociogramme-express et du recours aux ceintures de comportement puisque ces institutions, comme leurs conceptualisations, sont encore dans une phase balbutiante au sein de sa pratique (Bénévent et Mouchet, 2014, p. 116-141). Il faut enfin souligner toute la différence existant entre les groupes à visée thérapeutique conduits par un psychanalyste et les groupes institués dans une classe coopérative où personne ne prétend se situer à la place de l'analyste.

Deux autres acceptions du terme «groupe » peuvent aussi être relevées, I'une renvoyant aux groupes pédagogiques des enseignants membres du mouvement Freinet et l'autre évoquant plutôt le groupe sous l'influence de certains secteurs des sciences humaines. Il s'agit alors de 6 occurrences dans le premier cas et de 8 occurrences dans le second. Lorsqu'il est question des praticiens Freinet, on remarque que Fernand Oury utilise le terme pour désigner ses compagnons de travail, sans que l'appellation corresponde forcément à une désignation officielle dans le mouvement, puisqu'il écrit par exemple : « J'ai exposé, en mai dernier, aux camarades du Groupe Parisien de l'École Moderne, comment, de 1950 à 1954, j'ai tenté de résoudre les problèmes de la discipline et de la liberté » (Oury, 1955b, p. 26). Or l'instance dont il parle est I'IPEM qui est en effet le groupe parisien du mouvement Freinet, ainsi qu'il est encore indiqué aujourd'hui sur la page Facebook de I'IPEM ${ }^{10}$. Dans la même page que pour la précédente
10. https://fr-fr.facebook.com/ipem75 
citation, il utilise aussi des formulations qui semblent assimiler le mouvement lui-même à un groupe, comme lorsqu'il écrit : «Ici, nous sommes du métier et le problème des activités à la fois scolaires et enthousiasmantes est étudié, depuis plus de 25 ans, par des camarades du groupe Freinet ».

En quelques rares occasions, le groupe paraît être pensé non plus à partir d'un regroupement réel d'enfants ou d'adultes, mais sur un versant plus notionnel, avec de potentielles références aux sciences humaines. C'est en particulier le cas dans l'article de 1957 intitulé Le scolastisme où Fernand Oury indique qu'en étudiant les situations pathogènes générées selon lui par les grands établissements scolaires, «psychologues et sociologues découvriront alors que vie en troupeau et vie en groupe ne sont pas synonymes, qu'il est vain et finalement coûteux de vouloir éduquer "à la chaîne" » ou que « la classe autoritaire n'est pas un groupe vivant. L'œuvre collective est accidentelle. La collaboration s'appelle tricherie. Le groupement s'appelle bande » (Oury, 1957d, p. 8-9).

Mais ces usages du terme sont donc peu fréquents et ils apparaissent même parfois sous forme de citations, comme à l'occasion d'un article rendant compte d'un échange avec les docteurs Clément Launay, André Berge et Jean Oury à la suite d'un exposé de Fernand Oury montrant «l'inadaptation de l'école » lors d'une «soirée psycho-pédagogique » organisée par I'IPEM en 1957. Les propos cités sont par exemple: «Dans certains cas, l'expression libre publique doit favoriser, grâce à l'appui socialisant du groupe des phénomènes de catharsis efficaces» ou «plus il y aura d'échanges entre individus et entre groupes, plus on créera des conditions d'éducation valables » (Oury, 1957f, p. 8-10).

Peut-être pouvons-nous déjà percevoir ici une certaine ambivalence de la position de Fernand Oury vis-à-vis de l'usage de la théorisation ? Une position que Nicole Mosconi a fort bien relevée lorsqu'elle a fait la recension du livre «L'Année dernière, j'étais mort... » signé Miloud, paru en 1986. Voici ce que cette auteure écrivait alors : «s'élevant à juste titre contre un discours terroriste qui, pour maintenir chacun "à sa place", dénierait à l'instituteur, comme praticien et surtout comme "primaire", le droit à théoriser, [Fernand Oury] ne peut s'empêcher pourtant, face aux théorisations qu'il produit d'adopter une attitude très défensive » (Mosconi, 1988, p. 119). Elle relève notamment la phrase où Fernand Oury indique que, modeste instituteur, il pense avoir « pigé » certaines choses qu'il serait incapable de «comprendre » ou «d'expliquer rationnellement ». Une phrase en forme d'ironique dénégation qui « peut cependant être entendue comme l'expression d'une culpabilité inconsciente de qui a l'impression d'avoir usurpé une position interdite » (Mosconi, 1988, p. 119).

Un des effets de ce que nous désignons comme une attitude ambivalente est que si des références théoriques émergent ici ou là dans les textes de Fernand Oury, celles-ci apparaissent souvent sous une forme allusive apte à générer une «puissance évocatrice » (Blanchard-Laville et coll., 2005, 
p. 142) plutôt que selon des modalités susceptibles d'engager le lecteur à poursuivre plus avant l'étude des concepts abordés. Cette dernière remarque ne nous dispense toutefois pas de prêter attention à I'utilisation de quelques références conceptuelles dans les premiers écrits de Fernand Oury, ce qui sera l'objet de la partie qui suit.

\section{Quels points de tension dans les diverses références théoriques au groupe?}

Nous prendrons ici comme exemple le texte qui est à la fois le premier publié et le plus long parmi les articles retenus, «Une expérience de discipline démocratique dans un cours élémentaire de 40 élèves », paru en deux parties dans les numéros 2 et 5 de L'Éducateur en 1955, pour examiner la manière dont sont utilisés les repères théoriques dans les premiers écrits de Fernand Oury. Dans le domaine des «phénomènes psychologiques », c'est tout d'abord une référence à Jacques Lacan qui apparaît, sous forme d'une note de bas de page en conclusion de la première partie de l'article, immédiatement suivie d'une autre ${ }^{11}$ renvoyant cette fois à Jacob Levy Moreno : «Peut-être conviendrait-il d'étudier ici les phénomènes psychologiques d'identification, de désidentification [Lacan, 1950, La causalité psychique], les phénomènes microsociologiques "télé", etc. [Moreno, 1934, Who shall survive ?] ».

Ces mentions relativement allusives méritent d'être considérées de plus près. L'écrit de Lacan auquel Fernand Oury fait référence - et dont le titre exact est Propos sur la causalité psychique - a d'abord été publié dans Le problème de la psychogenèse des névroses et des psychoses, un ouvrage paru en 1950 aux éditions Desclée de Brouwer sous la direction de Lucien Bonnafé. Ce livre constituait les Actes du colloque de Bonneval qui s'était tenu du 28 au 30 septembre 1946 sur le thème «Causalité psychique des troubles mentaux ». Il comprenait les rapports présentés par Henri Ey, Jacques Lacan, Julien Rouart et Lucien Bonnafé ; celui de Lacan avait été prononcé le 28 septembre, en ouverture de la réunion. Contrairement à ce que l'appel de note renvoyant au terme désidentification pourrait laisser croire, ce sont les processus d'identification et les enjeux qui leur sont liés qui constituent le noyau de l'exposé du psychiatre psychanalyste, le vocable utilisé par Fernand Oury étant absent du texte signalé. Un texte où Lacan affirme par exemple: «nous croyons donc pouvoir désigner dans l'imago l'objet propre de la psychologie, exactement dans la même mesure où la notion galiléenne du point matériel inerte a fondé la physique [...] Une forme de causalité la fonde qui est la causalité psychique même : l'identification, laquelle est un phénomène irréductible, et l'imago est cette forme définissable dans le complexe spatio-temporel imaginaire qui a pour fonction de réaliser l'identification résolutive d'une phase psychique, autrement dit une métamorphose des relations de l'individu à son semblable » (Lacan, 1966 , p. 178).
11. Ces notes de bas de page sont indiquées entre crochets. 
Mais cette allusion discrète de Oury à l'imago lacanienne coexiste dans son article avec d'autres références. Certaines ont un caractère véritablement furtif, comme lorsqu'il est affirmé que «chaque équipe constitue une petite société réelle d'individus qui ne sont pas choisis pour être réunis et qui ne peuvent, sauf exception, se séparer. Plus tard, du reste, on ne choisit guère ses collègues, ses voisins d'autobus ou ses compagnons d'action » (Oury, $1955 b$, p. 28). Un tel énoncé évoque en effet très directement, par les exemples choisis qui sont les mêmes que dans le texte sartrien, le « collectif », la « sérialité » et le « groupe en fusion» théorisés par Sartre (1960) dans Critique de la raison dialectique, mais sans qu'il soit pour autant fait mention de cet ouvrage, sans que la dimension groupale soit clairement abordée sous l'éclairage des travaux du philosophe. Il faut d'ailleurs mentionner aussi que les premières notes de bas de page de l'article concernent la philosophie et le politique, dans un passage où Fernand Oury signale les difficultés rencontrées par l'enseignant qui tente de « voir plus clair» dans sa pratique : «Ce n'est pas facile pour l'instituteur empêtré dans la situation imposée par son métier: les coutumes, les règlements, I'Idée $[\mathrm{Au} \text { sens platonicien }]^{12}$ du métier, le rôle de $M$. I'Instituteur, toutes choses qui peuvent, en fin de compte, se ramener à la place qu'occupe l'École dans un État donné [Voir Lénine: L'État et la Révolution] ».

La référence à Jacob Levy Moreno à propos des «phénomènes microsociologiques "télé" » est, elle, un peu plus explicite et fait partie de références probablement plus familières pour Fernand Oury. En effet, la traduction française de Who shall survive?, dont la première édition aux États-Unis date de 1934, a été publiée en français en octobre 1954, sous le titre Fondements de la sociométrie. Fernand Oury a visiblement eu très rapidement connaissance de cet ouvrage. Les travaux de Moreno, considéré comme le père fondateur de la sociométrie et du psychodrame, ont été diffusés en France - notamment par Anne Ancelin Schützenberger - avant la traduction de cet ouvrage majeur. Fernand Oury n'est d'ailleurs pas le premier à avoir envisagé un intérêt de la sociométrie pour la pédagogie. En mai 1953, Gaston Mialaret proposait déjà des pistes d'utilisation « du sociogramme dans la conduite d'une classe » (Mialaret, 1953, p. 402), mais nous ne savons pas si Fernand Oury avait eu connaissance de ce texte. Plus tard, ces travaux ont été repris par des praticiens de la pédagogie institutionnelle comme Raymond Fonvieille (1999) ou Danièle Clairon-Vialon avec Aïda Vasquez (Oury et Vasquez, 1971, p. 513-530).

Moreno observe l'importance de la dimension affective dans les groupes et cherche à quantifier les liens d'attraction et de répulsion existant entre les individus dans le groupe. «Le sociogramme est une technique très simple qui consiste à interroger chacun des membres du groupe à partir d'un petit questionnaire d'évaluation des relations » (Lecourt, 2008, p. 67). Quant à la « fonction télé », Moreno la définit en lien avec ce qu'il nomme « l'atome social » (Moreno, 1954, p. 159-174), autrement dit ce qui constitue la « plus 
petite unité sociale » (Id., p. 160), « le réseau des interrelations d'un sujet, ses affinités électives, le noyau de relations autour d'un sujet » (Lecourt, 2008 , p. 68). Le télé « désigne une forme de co-empathie, de communication à distance entre les êtres. Ainsi l'atome social est composé d'un certain nombre de télés. Les individus sont plus ou moins pourvus de télé et ont des atomes sociaux plus ou moins riches » (Ibid.).

On peut se demander si de telles conceptions sont vraiment articulables avec les apports lacaniens, dont on sait qu'ils ont influencé Fernand Oury dès le début des années 1950. Toujours est-il qu'à travers ces diverses références, une certaine tension apparaît entre la dimension groupale abordée en fin d'article et les mentions faites de l'élève ou de l'enfant en tant qu'individu. Si la nécessité d'une dimension d'hétérogénéité dans l'équipe de travail est mise en avant (et l'on voit pointer là une notion fondamentale des conceptualisations propres à la psychothérapie institutionnelle), elle paraît être principalement au service de I'enrichissement des « inter-réactions » entre les enfants, pour reprendre les termes de l'auteur. Ce qui situerait l'attention portée à la gestion des groupes ou des équipes dans une tout autre perspective que ce que la référence au texte de Lacan pourrait laisser penser.

C'est ultérieurement, dans d'autres articles ou ouvrages, que Fernand Oury marquera sa proximité avec ce texte qu'il cite dès ses premiers écrits, quand il articulera plus précisément son approche pédagogique aux théorisations lacaniennes, notamment en ce qui concerne le processus de constitution du sujet ou les «interactions » entre les individus, entendues cette fois comme se situant dans le registre de l'inconscient. Il reviendra alors à ce qui était énoncé d'emblée dans le texte de Lacan, à travers des formulations qui lui sont propres: «Ainsi, point essentiel, le premier effet qui apparaisse de l'imago chez l'être humain est un effet d'aliénation du sujet. C'est dans l'autre que le sujet s'identifie et même s'éprouve tout d'abord » (Lacan, 1966).

La deuxième partie de l'article, publiée dans le numéro 5 de L'Éducateur ne procédera pas différemment. Cette fois, une seule référence est explicite (Poème pédagogique, d'Anton Makarenko), mais l'influence lacanienne est bien là, comme on peut la rencontrer avec la reprise des trois registres de I'Imaginaire, du Symbolique et du Réel (Lacan, 1958): «Nous voyons coïncider une liberté maximum sur le plan symbolique (expression libre) et une discipline très stricte sur le plan pratique. Il ne s'agit pas d'une mystification. Ici, liberté symbolique et discipline sont conditions l'une de l'autre. L'expression sur un mode symbolique de "I'imaginaire" permet l'acceptation du réel. L'organisation minutieuse d'un réel défavorable permet d'abord d'en changer le sens : au lieu de le subir sans savoir on prend des mesures pour le modifier, pour le dominer » (Oury, 1955d, p. 57).

Par la consultation des documents conservés à La Neuville, nous savons que Fernand Oury a assisté aux séminaires de Lacan sur « la relation d'objet » en 1956-57. On y trouve non seulement une version dactylographiée de ces 
séminaires, mais aussi les notes manuscrites prises par Fernand Oury au cours des séances (Archives de Fernand Oury à l'école de La Neuville, 2/38 et $2 / 39$ ).

\section{Conclusion}

Repérer certaines connexions entre la pensée de Fernand Oury au début de son œuvre et le contexte dans lequel il produit n'épuise pas la question de la conception du groupe chez cet auteur au début de sa pratique, ni celle des modalités de transmission sur ce sujet qui ont pu ensuite exister dans le courant dont il était co-fondateur. En ce qui concerne notre question de départ, celle de la faiblesse relative de l'approche psychanalytique groupale dans le champ de la pédagogie institutionnelle, nous devons bien admettre qu'elle demeure en grande partie posée. La complexité de la pensée de Lacan sur la question du groupe n'y est sûrement pas étrangère, comme nous le signalions en début d'article. De la même manière que René Kaës indique que «la position, plus que la pensée de J. Lacan a exercé une influence décisive dans toute son effervescence dans les groupes psychanalytiques à propos des groupes» (Kaës, 2013, p. 28), cette influence n'a pu que se faire sentir également chez les pédagogues qui se réclamaient de son enseignement.

Cette position, nous la trouvons notamment exposée dans un texte bien postérieur à l'époque des premiers écrits de Fernand Oury, mais que nous signalons quand même brièvement ici en ce qu'il nous paraît potentiellement éclairant. Voici un extrait de ce que Lacan écrivait en 1973 dans un article intitulé $L$ 'Étourdit : « le discours psychanalytique [...] est justement celui qui peut fonder un lien social nettoyé d'aucune nécessité de groupe. [...] je dirai que je mesure l'effet de groupe à ce qu'il rajoute d'obscénité imaginaire à l'effet de discours » mais aussi «qu'il est historiquement vrai que ce soit l'entrée en jeu du discours analytique qui a ouvert la voie aux pratiques dites "de groupe" et que ces pratiques ne soulèvent qu'un effet, si j'ose dire, purifié du discours même qui en a permis l'expérience. Aucune objection là, à la pratique dite "de groupe", pourvu qu'elle soit bien indiquée (c'est court) 》(Lacan, 1973, p. 31).

D'autre part, et ce point seul mériterait un nouveau travail, il faut bien constater que, du fait des liens importants entre psychothérapie et pédagogie institutionnelles, les matrices de théorisation les plus disponibles sont apportées par des psychiatres psychanalystes travaillant dans le champ de la psychose, alors que dans les classes dirigées par les praticiens Freinet, on ne trouve guère d'enfants psychotiques. Il n'est dès lors peut-être pas très étonnant que, dans l'intérêt porté aux élèves en difficulté, les pédagogues soient tentés d'explorer les phénomènes de groupe à l'aide de modèles théoriques du type de celui proposé par la sociométrie.

Enfin, comme nous l'avons mentionné plus haut, ce travail mériterait d'être complété par une recherche dans les archives de Raymond Fonvieille et 
prolongé par des investigations sur les publications postérieures à 1958. Outre ses ouvrages généralement connus, Fernand Oury a écrit de nombreux articles qui n'ont encore fait l'objet d'aucune recherche. Et puisque les livres fondateurs ont été co-signés, il serait sans nul doute intéressant de revenir à la thèse soutenue en 1966 par Aïda Vasquez qui proposait une Contribution à l'étude des relations humaines dans la classe. Quelle place était-elle faite dans cette recherche à la question du groupe et aux apports de la psychanalyse groupale? Voilà de nouvelles pistes d'investigation qui se dessinent au terme de cette tentative de restitution de résultats partiels concernant l'étude que nous avons menée.

\section{Références bibliographiques}

Amram, M. et d'Ortoli, F. (réalisateurs) (2010). Fernand Oury : un homme est passé [Film documentaire]. Vincennes : Frémeaux \& associés.

Anzieu, D. (1956). Le psychodrame analytique chez l'enfant. Paris : PUF.

Anzieu, D. et Martin, J.-Y. (1968). La dynamique des groupes restreints. Paris : PUF.

Bénévent, R. et Mouchet C. (2014). L'école, le désir et la loi. Fernand Oury et la pédagogie institutionnelle. Nîmes : Champ social.

Bion, W. R. (1961). Recherches sur les petits groupes. Paris : PUF, 1965.

Blanchard-Laville, C., Chaussecourte, P., Hatchuel, F. et Pechberty, B. (2005). Recherches cliniques d'orientation psychanalytique dans le champ de l'éducation et de la formation. Revue Française de Pédagogie, 151, 111-162.

Bullitt, W. et Freud, S. (1966/1990). Le Président Thomas Woodrow Wilson, portrait psychologique. Paris : Payot.

Carroy, J. (2005). L'étude de cas psychologique et psychanalytique (XIXe - début du $X X^{\mathrm{e}}$ siècle). Dans J.-C. Passeron, et J. Revel, (dir.), Penser par cas (p. 201-228). Paris : EHESS.

Caspard, P. et Caspard-Karydis, P. (2006). La presse d'éducation et d'enseignement. Dans T. Charmasson, (dir.), Histoire de l'enseignement, XIX ${ }^{e}-X X^{e}$ siècles. Guide du chercheur (p. 563-573). Paris : CTHS/INRP.

Devereux, G. (1995). Cléomène le roi fou. Paris : Aubier.

Dosse, F. (2007). Gilles Deleuze et Félix Guattari. Biographie croisée. Paris : La Découverte.

Dubois, A. (2011). Des premières monographies du courant psychanalytique de la pédagogie institutionnelle à la formation des enseignants aujourd'hui (thèse de doctorat non publiée). Université Paris Ouest Nanterre La Défense.

Dubois, A. et Geffard, P. (2013). Monographies et approche clinique d'orientation psychanalytique en Sciences de l'éducation. Congrès international Actualité de la recherche en éducation et en formation (AREF). Montpellier. En ligne : http://www.aref2013.univ-montp2.fr/cod6/?q=content/195-actualité-des-dispositifs -méthodologiques-pour-la- recherche-clinique-d'orientation

Fonvieille, R. (1958). Le congrès de Paris. L'Éducateur technologique, 16, 3-5.

Fonvieille, R. (1989). L'aventure du mouvement Freinet vécue par un praticien militant (1947-1961). Paris : Méridiens Klincksieck.

Fonvieille, R. (1998). Naissance de la pédagogie autogestionnaire. Paris : Anthropos.

Fonvieille, R. (1999). Face à la violence : participation et créativité. Paris : PUF.

Freud, S. (1939/1986). L'Homme Moïse et la religion monothéiste. Paris : Gallimard.

Geffard, P. (2010). Le tissage des liens professionnels dans la classe coopérative. Dynamiques psychiques en classe et en groupe de praticiens de la pédagogie 
institutionnelle (thèse de doctorat non publiée). Université Paris Ouest Nanterre La Défense.

Kaës, R. (2013). Un singulier pluriel. La psychanalyse à l'épreuve du groupe. Paris : Dunod.

Lacan, J. (1966). Écrits. Paris : Le Seuil.

Lacan, J. (1973). L'Étourdit, Scilicet, 4, 5-52.

Lamihi, A. (1994). De Freinet à la pédagogie institutionnelle, ou l'école de Gennevilliers. Vauchrétien : Ivan Davy.

Lebovici, S., Diatkine, R. et Kestemberg, E. (1952). Application de la psychanalyse à la psychothérapie de groupe et à la psychothérapie dramatique en France. Évolution psychiatrique, 3, 397-412.

Lecourt, E. (2008). Introduction à l'analyse de groupe. Rencontre psychanalytique de I'individuel et du social. Ramonville-Saint-Agne : Érès.

Mialaret, G. (1953). Sociométrie et pédagogie. Bulletin de psychologie, 7, 399-402.

Moreno, J.-L. (1954). Fondements de la sociométrie. Paris : PUF.

Mosconi, N. (1988). Pochet (Catherine), Oury (Fernand), Oury (Jean). - « L'Année dernière, j'étais mort... » signé Miloud. Revue française de pédagogie, 83, 116-119.

Oury, J. et Vasquez, A. (1967). Vers une pédagogie institutionnelle. Paris : Maspero.

Oury, J. et Vasquez, A. (1971). De la classe coopérative à la pédagogie institutionnelle. Paris : Maspero.

Rouchy, J.-C. (2009). Transmission intergénérationnelle dans le groupe d'appartenance. Dialogue, 186, 149-160.

Rousso, H. (2002). Analyse de I'histoire. Analyse de I'historien. Espaces Temps, 81-82, 126-134.

Sartre, J.-P. (1960). Critique de la Raison dialectique. Théorie des ensembles pratiques [vol 1] - Questions de méthode (précédé de). Paris : Gallimard.

Savoye, A. (2004). L'éducation nouvelle en France, de son irrésistible ascension à son impossible pérennisation (1944-1970). Dans A. Ohayon, D. Ottavi, et A. Savoye (éds.), L'éducation nouvelle, histoire, présence et devenir (p. 235-269). Bern : Peter-Lang.

Tosquelles, F. (2014). L'enseignement de la folie. Paris : Dunod.

Vasquez, A. (1966). Contribution à l'étude des relations humaines dans la classe. Étude des classes primaires actives françaises. Pédagogie institutionnelle (thèse de doctorat non publiée). Université de la Sorbonne, Paris.

Voldman, D. (1993). La place des mots, le poids des témoins. Dans Écrire l'histoire du temps présent. En hommage à François Bédarida (p. 123 à 131). Paris : C.N.R.S.

\section{Articles publiés par Fernand Oury entre 1955 et 1958}

Oury, F. (1955a). À propos du matériel scolaire. L'Éducateur, 27-28, 7-8.

Oury, F. (1955b). Une expérience de discipline démocratique dans un cours élémentaire de 40 élèves. L'Éducateur, 2, 25-28.

Oury, F. (1955c). Le mobilier des classes actives. Éducation nationale, 28, 11-12.

Oury, F. (1955d). Une expérience de discipline démocratique dans un cours élémentaire de 40 élèves (suite). L'Éducateur, 5, 55-58.

Oury, F. (1957a). Le scolastisme. L'Éducateur, 16, 7-9.

Oury, F. (1957b). Chronique de la fosse aux ours, journal d'un instituteur de banlieue. L'Éducateur, 16, 10-13.

Oury, F. (1957c). La classe moderne dans le groupe urbain, Compte-rendu d'une enquête dans le groupe parisien (22 novembre 1956), suivi de notes en marge d'un rapport. L'Éducateur, 20, 17-23

Oury, F. (1957d). Le scolastisme. Revue pratique de psychologie, de la vie sociale et d'hygiène mentale, 2, 14-17. (Texte déjà publié dans L'Éducateur, 16) 
Oury, F. (1957e). Chronique de la Fosse aux ours : Du Sang ! Du Sang ! L'Éducateur, 3, 7-8.

Oury, F. (1957f). D’une soirée psychopédagogique. L'Éducateur d'île-de-France, 9, 8-9.

Oury, F., Michaud, G. (1957g). Exposition de travaux des hôpitaux psychiatriques. L'Éducateur d'île-de-France, 10, 11-12.

Oury, F. (1958a). Chronique de la fosse aux ours. Des sanctions. L'Éducateur d'île-deFrance, 12, 5-9. (Texte publié dans Techniques de vie en 1961)

Oury, F. (1958b). Pourquoi ne parle-t-on pas des classes Freinet à Paris ? L'Éducateur d'île-de-France, 14, 3-4.

Arnaud Dubois

MCF en sciences de l'éducation

Laboratoire EMA

Université de Cergy-Pontoise

Patrick Geffard

MCF en sciences de l'éducation

Laboratoire CIRCEFT, CLEF-apsi

Université Paris 8 Vincennes-St-Denis

Pour citer ce texte :

Dubois, A. et Geffard, P. (2015). Fernand Oury, les

groupes de la classe coopérative et l'approche

psychanalytique (1955-1958). Cliopsy, 14, 37-55. 